\title{
NATURE VS. NURTURE DEBATE ON TNO CARBONS: CONSTRAINTS FROM RAMAN SPECTROSCOPY
}

\author{
R. Brunetto ${ }^{1}$
}

\begin{abstract}
We compare spectroscopic data of irradiated laboratory analogs with those of an interplanetary dust particle of cometary origin. We investigate if this comparison can help constraining the origin of carbonaceous materials on small icy bodies in the outer Solar System (TNOs, Centaurs, etc.). We suggest that Raman spectroscopy can help in interpreting the observed heterogeneity of the extraterrestrial carbonaceous component and in constraining the irradiation dose accumulated in space.
\end{abstract}

\section{Introduction}

Complex organic materials on small outer Solar System icy bodies probably include a primary native component accreted during the formation of planetesimals ("nature"), and a secondary component that is a by-product of (cosmic and/or solar wind) ion and photon irradiation of simpler C-bearing volatile ices ("nurture"). The debate of nature vs. nurture has been recently summarized by Dalle Ore et al. (2011). An example of an object which shows the presence on its surface of a red carbonaceous component (nature?) and of a black component (nurture?) has been discussed by Guilbert et al. (2009). Experimental studies can simulate some of these processes and provide interesting inputs to this debate on the origin of the carbonaceous component of trans-Neptunian Objects (TNOs) and Centaurs.

\section{Micro-Raman spectra of a cometary IDP}

Chondritic porous (CP) IDPs probably originated from comets, or P-type or D-type asteroids. They are good analogs of the refractories (carbons, silicates) possibly present on small outer Solar System icy bodies. A CP-IDP of cometary origin (IR spectra match the small silicate grains emission of comet Hale-Bopp)

1 Institut d'Astrophysique Spatiale, CNRS, UMR-8617, Université Paris-Sud, Bât. 121, 91405 Orsay Cedex, France 
provided by NASA was analyzed with IR, Raman, and FESEM-EDX, in the framework of the analysis of cometary materials performed by the "Astrochimie et Origines" team in Orsay, in collaboration with SMIS-SOLEIL and Università di Napoli "Parthenope".

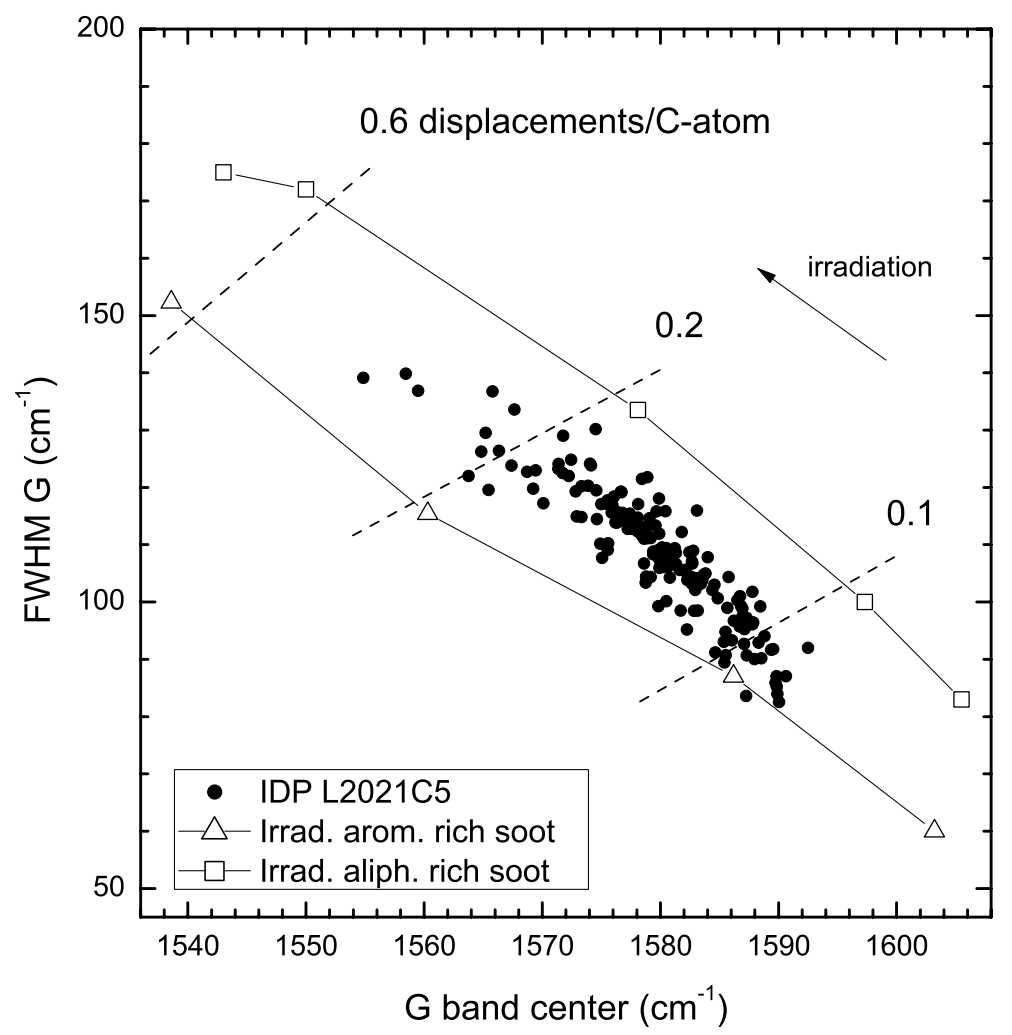

Fig. 1. G band full width at half maximum (FWHM) as a function of G band peak position, for IDP L2021C5 (Brunetto et al. 2011) and irradiated aliphatic-rich and aromaticrich soot (Brunetto et al. 2009). An arrow marks the evolutionary path due to ion irradiation.

Raman spectra have evidenced strong heterogeneity of the carbonaceous structures with different degrees of order (Brunetto et al. 2011). The combination of the three techniques indicates that the particle experienced only mild flash heating on atmospheric entry. Raman spectroscopy is sensitive to the carbon structure and to its degree of order. It has been used to characterize the organic matter of meteorites and IDPs (e.g. Wopenka 1988; Matrajt et al. 2004; Quirico et al. 2005; Busemann et al. 2007), and organics captured from comet 81P/Wild 2 (e.g. Rotundi et al. 2008; Muñoz-Caro et al. 2008). 


\section{Comparison with irradiated laboratory analogs}

In Figure 1 we compare spectroscopic data of irradiated soot (laboratory analogs of cosmic dust, Brunetto et al. 2009) with those of IDP L2021C5. This preliminary comparison can help constraining the origin of TNO carbonaceous materials. Baratta et al. (2004) found an upper limit to the highest dose accumulated by IDPs due to ion irradiation in the interplanetary medium: about 0.5 displacements per $\mathrm{C}$ atom, compatible with the exposure time of these particles.

We find that about $80 \%$ of carbons in IDP L2021C5 have upper limit dose between $0.1-0.2$ displacements per C-atom. Only about $10 \%$ of carbons can be compatible with high irradiation dose. Further investigation is needed to determine whether the observed heterogeneity of the extraterrestrial carbonaceous component is primordial or an effect of irradiation during residence in interplanetary medium. In any case, we confirm that Raman spectroscopy of relevant extraterrestrial carbons gives interesting and complementary (to other techniques) information that can help in interpreting the observed heterogeneity of the extraterrestrial component and in constraining the dose accumulated in space.

We are grateful to the "Astrochimie et Origines" team and the SMIS-SOLEIL team. This Research has been supported by the French "Agence Nationale de la Recherche".

\section{References}

Baratta, G.A., Mennella, V., Brucato, J.R., et al., 2004, JRSp, 35, 487

Brunetto, R., Pino, T., Dartois, E., et al., 2009, Icar, 200, 323

Brunetto, R., Borg, J., Dartois, E., et al., 2011, Icar, 212, 896

Busemann, H., O'D. Alexander, C.M., \& Nittler, L.R., 2007, M\&PS, 42, 1387

Dalle Ore, C.M., Fulchignoni, M., Cruikshank, D.P., et al., 2011, A\&A, 533, A98

Guilbert, A., Barucci, M.A., Brunetto, R., et al., 2009, A\&A, 501, 777

Matrajt, G., Borg, J., Raynal, P.I., et al., 2004, A\&A, 416, 983

Muñoz-Caro, G.M., Dartois, E., \& Nakamura-Messenger, K., 2008, A\&A, 485, 743

Quirico, E., Borg, J., Raynal, P.-I., Montagnac, G., \& d'Hendecourt, L., 2005, P\&SS, 53, 1443

Rotundi, A., Baratta, G.A., Borg, J., et al., 2008, M\&PS, 43, 367

Wopenka, B., 1988, E\&PSL, 88, 221 